\title{
Animal Model of Antibiotic Induced Gut Microbiota Dysbiosis $^{\dagger}$
}

\author{
Christine A. Butts 1,*, Gunaranjan Paturi ${ }^{2}$, Halina Stoklosinski ${ }^{1}$, Sheridan Martell ${ }^{1}$, \\ Duncan Hedderley ${ }^{1}$ and Elizabeth Carpenter ${ }^{3}$ \\ 1 New Zealand Institute for Plant and Food Research Ltd., Palmerston North 4474, New Zealand; \\ Halina.Stoklosinski@plantandfood.co.nz (H.S.); Sheridan.Martell@plantandfood.co.nz (S.M.); \\ Duncan.Hedderley@plantandfood.co.nz (D.H.) \\ 2 Plant and Food Research, Auckland 1025, New Zealand; Gunaranjan.Paturi@plantandfood.co.nz \\ 3 Dairy Goat Co-operative (NZ) Ltd., Hamilton 3240, New Zealand; Liz.Carpenter@dgc.co.nz \\ * correspondence: Chrissie.Butts@plantandfood.co.nz \\ + Presented at the 2018 Nutrition Society of New Zealand Annual Conference, Auckland, New Zealand, \\ 28-30 November 2018.
}

Published: 5 March 2019

Background: The gut harbors a diverse ecosystem consisting predominantly of bacteria. The gut microbiota and host tissues closely interact and disruption of this relationship causes intestinal dysbiosis which has been linked to asthma, inflammatory bowel disease and metabolic disorders. Gastrointestinal dysbiosis has been induced experimentally using antibiotics but few studies have compared the effects of different antibiotics or length of exposure. This study compared different antibiotic treatments on gut microbiota in newly weaned rats to establish a small animal model of gastrointestinal dysbiosis.

Methods: Male Sprague-Dawley rats (3 weeks old) were randomly allocated to the experimental treatments $(n=6)$ : control (no antibiotic), amoxicillin or antibiotic mixture (amoxicillin, gentamicin, metronidazole). The rats were given ad libitum access to food and water throughout the study. The antibiotics were administered in the drinking water for 7 and 14 days. The rats were euthanased at the end of the experimental period and the gut contents and tissues removed for analysis. These procedures were approved by the AgResearch Grasslands Animal Ethics Committee (Application 13948).

Results: Amoxicillin treatment resulted in significantly lower numbers of Bifidobacterium spp. in the caecum, colon and faeces, while higher numbers of Lactobacillus spp. were detected in the caecum of these rats. The antibiotic mixture significantly decreased the Bifidobacterium spp., Lactobacillus spp. and total bacteria in the caecum, colon and faeces. Changes induced by the antibiotics were greater when the rats were treated with the antibiotic mixture than with amoxicillin, and greater after 14 days than after 7 days of antibiotic treatment.

Conclusions: Intestinal dysbiosis was successfully established under these experimental conditions. Future studies will characterize the effect of foods and diet on gut microbiota dysbiosis.

(C) 2019 by the authors. Licensee MDPI, Basel, Switzerland. This article is an open access article distributed under the terms and conditions of the Creative Commons Attribution (CC BY) license (http://creativecommons.org/licenses/by/4.0/). 